\title{
ĐÁNH GIÁ CHẤT LỰ̛̣̂ GIẢNG DẠY CỦA GIẢNG VIÊN KHỐI KINH TẾ TẠI TRƯờnG ĐẠI HỌC CÔNG NGHIỆP THÀNH PHỐ HỒ CHÍ MINH - BÀ̀ CHÚNG PHỤC VỤ KIỂM ĐỊNH AUN
}

\author{
HUỲNH TẤN DŨNG, LÊ THI HẢI BÌNH, HOÀNG ĐÌNH VUI, TRÀN THỨ BA \\ Khoa Kế toán - Kiểm toán, Truờng Đại học Công nghiệp thành phố Hồ Chí Minh \\ huynhtandung@iuh.edu.vn
}

Tóm tắt. Trong quá trình hội nhập giáo dục với các nước trong khu vực và thế giới, nhiều trường đại học trong nước đi vào chặn đường kiểm định chất lượng để nâng cao chất lượng đào tạo. Trường Đại học Công nghiệp thành phố Hồ Chí Minh (IUH) cũng đang đi trên con đường này và đang chuẩn bị để việc kiểm định chất lượng đào tạo theo chuẩn khu vực AUN (ASEAN University Network). Trong kiểm định theo chuẩn AUN, việc đánh giá chất lượng giảng dạy của giảng viên là hết sức cần thiết vì đội ngũ giảng viên là một trong những nhân tố quan trọng quyết định đến chất lượng đào tạo. Để góp phần cho việc kiểm định AUN thành công ở các khoa thuộc khối ngành Kinh tế của IUH, bài báo thực hiện khảo sát và đánh giá chất lượng giảng dạy của các giảng viên các khoa thuộc khối Kinh tế tại IUH từ đó đưa ra những giải pháp để nâng cao chất lượng giảng dạy cho các khoa này.

Bài báo sử dụng phương pháp định tính kết hợp định lượng, công cụ khảo sát là bảng câu hỏi. Nhóm nghiên cứu tiến hành khảo sát 902 sinh viên thuộc 4 khoa trong khối ngành Kinh tế của $\mathrm{IUH}$, từ đó phân tích kết quả khảo sát sinh viên và kết quả học tập của môn học được khảo sát tương ứng với từng giảng viên giảng dạy. Kết quả khảo sát phản ánh kết quả học tập không tỷ lệ thuận với mức độ hài lòng của sinh viên mặc dù mức độ hài lòng của sinh viên về chất lượng giảng dạy của giảng viên thuộc các khoa khối Kinh tế ở mức cao, cụ thể các giá trị về mức độ hài lòng nằm từ 3.77 đến 4.50 . Từ kết quả nghiên cứu trên, bài báo rút ra các kết luận về mối tương quan giữa mức độ hài lòng của sinh viên và kết quả học tập đạt được, nghiên cứu cũng đề xuất các giải pháp để cải tiến chất lượng giảng dạy của giảng viên, đồng thời kết quả nghiên cứu của bài báo cũng giúp các trưởng khoa khối Kinh tế, Ban giám hiệu có cái nhìn sâu sắc và đúng đẳn hơn trong nâng cao chất lượng giảng dạy của các khoa trong khối kinh tế của IUH.

Từ khóa: Chất lượng giảng dạy, đánh giá chất lượng giảng dạy, kiểm định AUN, trường Đại học Công nghiệp thành phố Hồ Chí Minh.

\section{ASSESSING THE QUALITY OF ECONOMIC LECTURERS IN THE INDUSTRY UNIVERSITY OF HOCHIMINH - EVIDENCE FOR AUN ASSESSMENT}

\begin{abstract}
In the process of integrating education with other countries in the region and the world, many universities in Vietnam have conducted to accredite education programme in order to improve the quality of teaching and training. The Industry University of in Ho Chi Minh is also on this way and is preparing for accreditation of AUN-QA (ASEAN University Network-Quality Assurance). In AUN-QN criteria, assessing the teaching quality of lecturers is very necessary because lecturers are one of the important factors that determine the quality of teaching and training. In order to contribute to successful AUN-QN assessment at the Faculty of Economics in the Industry University of in Ho Chi Minh, the paper examines and evaluates the teaching quality of lecturers in the Faculty of Economics then suggests solutions to improve the teaching quality in these departments.

The paper uses the qualitative method and quantitative method, questionnaire used to survey. The paper examines 902 students from 4 faculties in IUH's Economics Department. The article analyzes the results student satifaction and study results of each subject surveyed corresponding to each lecturer. Survey results show that student satifaction is not proportional to summative cores, although level of satisfaction of students on the quality of teaching of the lecturers in the Faculty of Economic sector at a high level, mean of satisfaction is between 3.77 and 4.50. Based on the results of the study, the paper suggests solutions to change the methodology of assessing the teaching quality of lecturers, and the results of the study also help
\end{abstract}


the heads of the Faculty of Economics, the Board of headmaster have look insight in improve the teaching quality of faculty in the economic sector of the University of Industry Ho Chi Minh City.

Keywords: Teaching quality, teaching quality assessment, AUN-QA, Industry University of Ho Chi Minh.

\section{Giới thiệu}

Chất lượng giảng dạy của giảng viên cả nước nói chung và ở thành phố Hồ Chí Minh nói riêng đề được các trường đại học quan tâm, đặc biệt là trong quá trình cả nước đang trong giai đoạn hội nhập về giáo dục. Bằng chứng là hiện nay rất nhiều trường đại học đang tiến hành kiểm định theo các tiêu chuẩn quốc tế hoặc khu vực như chuẩn ABET, AUN. Một trong những chỉ tiêu quan trọng trong việc kiểm định đó là chất lượng giảng dạy của giảng viên. Tuy nhiên việc đánh giá đúng chất lượng giảng dạy của giảng viên hiện nay gặp nhiều khó khăn và đôi lúc còn làm sai lệch kết quả về chất lượng giảng dạy của giảng viên. Theo PGSTS. Nguyễn Thị Thu Trang, việc kiểm định, đánh giá chất lượng đội ngũ giảng viên hiện nay dựa vào nhiều qui định (tiêu chí) chung như: bằng cấp, trình độ chuyên môn, thâm niên công tác, điểm $\mathrm{NCKH}$, đánh giá phản hồi của sinh viên, kết quả thanh tra nhà giáo, đánh giá xếp loại thi đua của đơn vị, tổ chức thao giảng... Nhưng thực tế việc đánh giá này vẫn rất hình thức và kém hiệu quả. Đánh giá hay kiểm định chất lượng giảng dạy là nhiệm vụ quan trọng và cần thiết. Giảng viên là những người lao động đặc biệt nên cũng cần có sự đánh giá kết quả lao động công bằng để nỗ lực phấn đấu đạt năng suất và kết quả cao hơn. Đánh giá đúng, phù hợp sẽ thúc đẩy sự tiến bộ và ngược lại [1]. Theo Thi Lan Phuong Pham (2012) [2], việc cải cách giáo dục của chính phủ Việt Nam giúp về chính sách và hệ thống giáo dục đặt ra yêu cầu với các trường đại học là học phải nâng cao chất lượng giảng dạy của giảng viên. Trong nghiên cứu này tác gỉa nghiên cứu chủ yếu các chính sách tác động đến nhân chất lượng giảng dạy của giảng viên và vai trò của chính phủ trong việc nâng cao chất lượng giảng dạy. Harry G. Murray (2006) [5], nghiên cứu việc đánh giá của sinh viên trong hoạt động giảng dạy của giảng viên và đánh giá kết quả học tập cuối kỳ của sinh viên cung cấp nhiều thông tin để cải tiến chất lượng giảng dạy. Lê Đình (2008), việc đánh giá hoạt động giảng dạy của giảng viên hay có thể gọi là đánh giá giảng dạy (teaching evaluation) là một yêu cầu không thể thiếu được đối với một cơ sở đào tạo nhằm đảm bảo và nâng cao chất lượng giáo dục [4]. Suryaman (2018) [6], hoạt động của giảng viên liên quan đến chất lượng trường đại học. Hoạt động của giảng sẽ ảnh hưởng đến chất lượng và khả năng mang tính bền vững của một tổ chức, trong trường hợp này là trường đại học. Jian Xiao and Stephen Wilkins (2015) [7], sự hài lòng của sinh viên đã trở thành một khái niệm quan trọng trong giáo dục đại học bởi vì sinh viên đang trả học phí cao hơn và ngày càng thấy mình là khách hàng và bởi vì sự hài lòng thường được sử dụng như một chỉ số về chất lượng. Trong nghiên cứu này Jian Xiao and Stephen Wilkins muốn để kiểm tra ảnh hưởng của cam kết giảng viên về nhận thức của sinh viên về chất lượng giảng dạy và sinh viên sự thỏa mãn. Nghiên cứu đã chỉ ra hoạt động của giảng viên có tác động đến sự hài lòng và kết quả đạt được của sinh viên. Jacqueline Douglas \& Alex Douglas (2006) [8], thực hiện so sánh bằng bảng câu hỏi phản hồi của sinh viên như một phương tiện đánh giá chất lượng dạy và học và cung cấp một phương tiện để cải tiến liên tục. Theo Drs. H. Akbar Ali, M.Si (2015) [9], hệ thống đảm bảo chất lượng là một hệ thống quản lý để chỉ đạo và kiểm soát tổ chức trong việc đưa ra quy trình, thủ tục chính sách, mục tiêu, kế hoạch và chất lượng và cũng thực hiện cải tiến liên tục, bao gồm cấu trúc tổ chức, trách nhiệm, quy trình, quy trình và tài nguyên được sử dụng để đạt được tiêu chuẩn đã được giải quyết dựa trên các bên liên quan và nhu cầu và yêu cầu của tổ chức. Đảm bảo chất lượng trong một tổ chức là nhu cầu nội bộ và bên ngoài. Đảm bảo chất lượng là một nhiệm vụ thường xuyên và có được thực hiện liên tục và nó không phải là một hoạt động đặc biệt. Năng lực của giảng viên là giảng viên khả năng thực hiện nghĩa vụ của mình một cách thích hợp. Trong trường hợp này, thẩm quyền của giảng viên sẽ có tác động đến thành tích và thành tích của giảng viên và sẽ đóng góp cho chất lượng của một trường đại học và cựu sinh viên của trường. Hiệu suất của một giảng viên sẽ tạo ra một tác động lớn đến việc thực hiện giáo dục một cách hiệu quả.

Như vậy có phải sự hài lòng của sinh viên đối với giảng viên tỷ lệ thuận với kết quả học tập của sinh viên hay không? Có trường hợp ngược lại hay không? Hay mức độ hài lòng của sinh viên đối với hoạt động của giảng viên không có liên quan gì đến kết quả học tập của sinh viên. Làm sao để biết được chất lượng giảng dạy của khoa mình ở đâu, chất lượng lượng giảng dạy của giảng viên ở mức nào? Ở $\mathrm{IUH}$, các khoa dựa vào mức độ hài lòng của sinh viên để đánh giá chất lượng giảng viên mà ít quan quan tâm đến sinh viên và giảng viên đạt được những gì trong quá trình học tập. Phát hiện ra vấn đề chưa hợp lý trong việc đánh giá 
chất lượng giảng dạy của giảng viên của các khoa trong khối kinh tế của IUH hiện nay, bài báo tiến hành nghiên cứu định lượng quy trình đánh giá gồm 2 giai đoạn: khảo sát sự hài lòng của người học đối với người dạy; đo lường mối quan hệ giữa sự hài lòng của người học và kết quả học tập của từng môn học được giảng dạy bởi giảng viên cụ thể đã được khảo sát bằng công cụ thống kê.

Việc nghiên cứu đánh giá chất lượng giảng dạy của giảng viên đối với sinh viên tại trường Đại học Công Nghiệp thành phố Hồ Chí Minh bằng quy trình 2 giai đoạn như nêu trên nằm cung cấp mức độ hài lòng của sinh viên về hoạt động giảng dạy của giảng viên và cũng nhằm kiểm định lại các việc đánh giá chất lượng giảng dạy dựa trên mức độ hài lòng của sinh viện có đánh tin cậy không. Kết quả nghiên cứu này sẽ cung cấp các bằng chứng đáng tin cây để từ đó, trường Đại học Công Nghiệp thành phố Hồ Chí Minh có thể đưa ra những biện pháp, cách thức đúng đắn nhằm cải tiến chất lượng giảng dạy của giảng viên nói riêng, chất lượng đào tạo của nhà trường nói chung trong quá trình đạt kiểm định chất lượng theo tiêu chuẩn AUN. Laura Goe (2007) [3], chất lượng giảng viên được định nghĩa và đo lường bằng nhiều cách. Một đồng thuận phổ biến rằng vấn đề chất lượng giảng viên thể hiện một mặt của thành quả sinh viên, nhưng không có sự đồng thuận rõ ràng rằng mặt nào của chất lượng giảng viên ảnh hưởng nhất hoặc thậm chí không có một định nghĩa chung về chất lượng giảng viên. Một lý do của sự định nghĩa khó khăn này là vì chất lượng giảng viên cần được định nghĩa khác nhau cho các mục đích khác nhau. Ví dụ, tiêu chí chỉ ra chất lượng liên quan đến quyết định tuyển dụng sẽ khác với tiêu chí dùng để bổ nhiệm vị trí, khen thưởng làm việc tốt, hoặc nhận diện và hỗ trợ giảng viên nỗ lực. Ngoài sự đóng góp giảng viên đối với thành quả sinh viên, chất lượng giảng viên có thể thể hiện qua những mặt như: bằng cấp và kinh nghiệm phù hợp cho cấp bậc và môn học, nhận được sự kỳ vọng cao của sinh viên, tạo được môi trường học tập khuyến khích sinh viên tham gia vào hoạt động học tập, mong muốn giúp cho sinh viên đạt được cấp độ cao, có khả năng khuyến khích sinh viên cá biệt tham gia lớp học, mặc dù điểm số của những sinh viên này không tốt, có kỹ năng tốt để hướng dẫn giáo viên mới, sã̃n sàng làm việc với sinh viên với các nhu cầu đặc biệt.

Cũng theo Laura Goe (2007), chất lượng giảng dạy và chất lượng giảng viên là hai khia cạnh khác nhau. Chất lượng giảng viên được xem là một bộ các tiêu chí đầu vào như bằng cấp, điểm số, chứng chỉ... để chỉ ra rằng họ có thể thành công trong lớp học. Trong khi, chất lượng giảng dạy là chất lượng những gì họ làm thực tế trên lớp học. Vì vậy, chúng có mối liên kết với nhau và nhiều khi được gộp thành một, do đó, giả định rằng chất lượng giảng viên đảm bảo chất lượng giảng dạy và chất lượng giảng dạy là kết quả đầu ra của chất lượng giảng viên. Chất lượng giảng dạy được chia ra thành hai vấn đề: một là nhiệm vụ giảng dạy của giảng viên đó là những gì giảng viên làm cho sinh viên, hai là thành quả của sinh viên là những gì sinh viên học được thông qua sự thúc đẩy của giảng viên.

Ở Việt Nam một số đề tài nghiên cứu mới chỉ dùng lại ở sự đo lường mức độ hài lòng của người học đối với người dạy mà chưa có sự liên hệ giữa sự hài lòng và và chất lượng giảng dạy (kết quả cuối cùng mà người học đạt được). Qua các nghiên cứu ở trên, việc đánh giá chất lượng giảng dạy của giảng viên là một vấn đề khoa học và nhiều nhà nghiên cứu trên thế giới quan tâm. Tuy nhiên ở Việt Nam nhóm tác giả chưa tìm thấy đề tài nghiên cứu hay bài báo nào công bố việc nghiên cứu định lượng về chất lượng giảng viên và mức độ tác động của hoạt động giảng dạy của giảng viên đến kết quả học tập của sinh viên.

Theo nhóm tác giả, việc nâng cao chất lượng giảng dạy đòi hỏi phải căn cứ vào các dữ liệu thực nghiệm về tình hình thực tế giảng dạy và học tập trong lớp học của sinh viên. Vì vậy bài báo này thực hiện nghiên cứu thực nghiệm tại IUH để bồ sung thêm các kết luận, các giải pháp và các kiến nghị đã được các tác giả trước đưa ra.

\section{Thực trạng đánh giá chất luọng giảng dạy của giảng viên tại trường Đại học Công nghiệp thành phố Hồ Chí Minh}

Trong quá trình chuẩn bị kiểm định $\mathrm{AUN}$, nhóm tác giả có trao đổi với lãnh đạo khoa của một số trường đại học ở thành phố Hồ Chí Minh, hiện nay các khoa cũng chưa có xây dựng riển bộ tiêu chí đánh giá chất lượng giảng dạy của giảng viên mà chỉ dựa vào mức độ hài lòng của sinh viên để làm căn cứ đánh giá chất lượng hoạt động giảng dạy của giảng viên. IUH cũng đang thực hiện như vậy. Hiện nay các khoa Kinh Tế của IUH dựa vào kết quả khảo sát môn học cuối khóa của sinh viên làm một trong những tiêu chí đánh giá chất lượng giảng dạy của giảng viên.

Tuy nhiên theo nhóm tác giả kết quả này mới chỉ đánh giá mức độ hài lòng của sinh viên đối với giảng viên chứ chưa đánh giá được chất lượng giảng dạy của giảng viên. Nếu chỉ dùng kết quả khảo sát này mà chura có sụ chứng minh mối liên hệ giữa sụ hài lòng và kết quả dạt được của người học thì việc đánh giá chất 
lương dạ hoc của người dạy là chua chính xác. Bằng chứng có nhiều lớp có điểm thường kỳ, giữa kỳ rất cao nhưng điểm cuối kỳ thi theo đề chung thì lại rất thấp. Theo thống kê số liệu của các khoa Kinh tế trong $\mathrm{IUH}$, các lớp có điểm thường kỳ, giữa kỳ cao sinh viên đều đánh giá mức độ hài lòng đối với giảng viên cao vì đa số các khoa thực hiện khảo sát khi chưa kết thúc môn học. Lúc này sinh viên đánh giá hoạt động giảng dạy của giảng viên chưa có sự liên hệ với kết quả mà sinh viên đạt được. Như vậy hoạt động giảng dạy của giảng viên đóng góp vào kết học tập của sinh viên như thế nào, và mức đóng góp của từng hoạt động là bao nhiêu thì hiện nay IUH chưa có nghiên cứu cụ thể và các khoa sắp kiểm định AUN năm 2019 cũng chưa biết.

\section{Phương pháp nghiên cứu}

Bài báo này dựa vào lý luận nghiên cứu của tác giả Laura Goe (2007) để thực hiện đo lường chất lượng giảng dạy của giảng viên khối Kinh tế tại IUH. Lý luận của mô hình như sau:

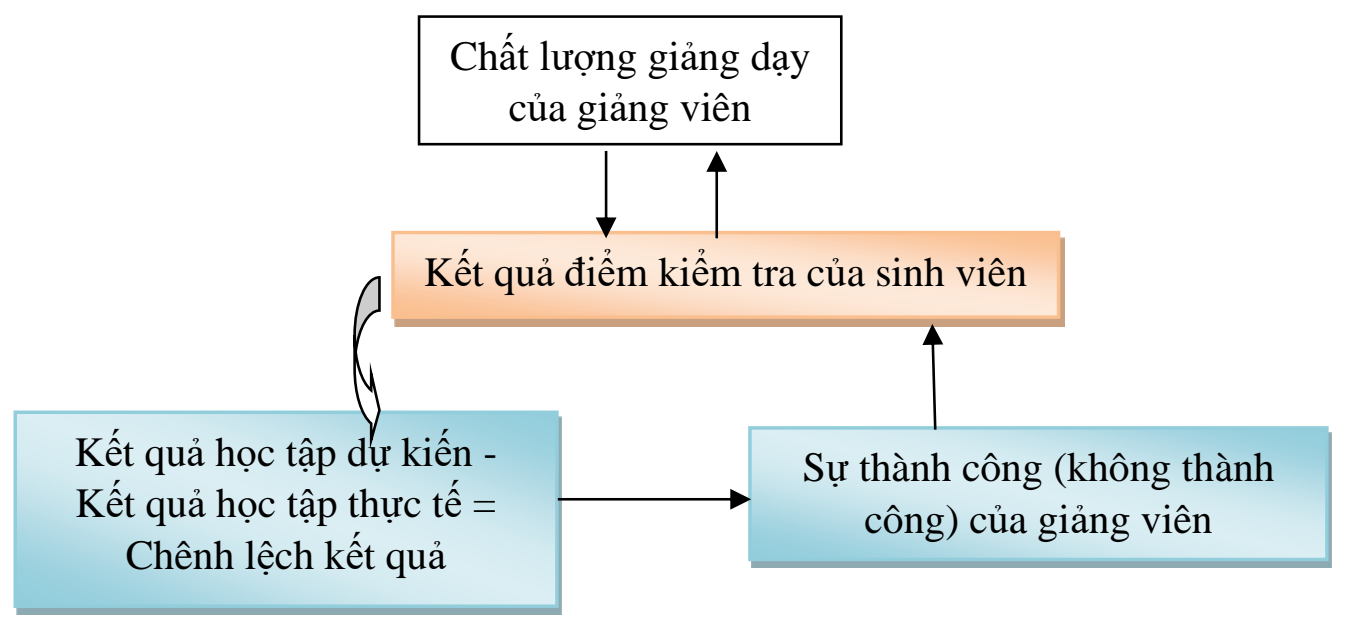

Nguồn: tổng hợp tù̀ nghiên cứu của Laura Goe 2007 Theo mô hình này kết quả điểm kiểm tra của sinh viên được xem như chỉ số đo lường chất lượng giảng viên. Sự chênh lệch kết quả điểm kiểm tra thực tế và điểm kiểm tra dự kiến của sinh viên thể hiện sự thành công (không thành công) của giảng viên.

Thông qua việc tổng hợp kết quả của các nghiên cứu của các tác giả trước đó, bài báo sử dụng phương pháp khảo sát định luợng, thống kê phân tích từ đó phân tích, đánh giá chất lượng giảng dạy của giảng viên khối ngành Kinh Tế tại trường đại học Công Nghiệp thành phố Hồ Chí Minh.

Cách thức thực hiện

Bước 1: Thực hiện xây dựng bảng câu hỏi

Bước 2: Tổ chức khảo sát

Bước 3: Nhập dữ liệu khảo sát

Bước 4: Nhập dữ kết quả học tập thực tế theo từng sinh viên theo từng cột điểm thường kỳ, giữa kỳ và cuối kỳ.

Bước 5: Thực hiện các phân tích thống kê theo từng giảng viên.

Bảng câu hỏi và thang đo

Nghiên cứu định tính được tiến hành khảo sát ý kiến của một số chuyên gia về giáo dục đại học như Phó hiệu trưởng phụ trách đào tạo IUH, trợ lý hiệu trưởng IUH, Trưởng khoa và một số đồng nghiệp là giảng viên IUH. Đồng thời, thực hiện phát phiếu khảo sát (180 phiếu) đối tượng là sinh viên trường đại học Công Nghiệp thành phố Hồ Chí Minh (chủ yếu là sinh viên năm 3, 4 gồm cả hệ liên thông). Sau nhiều lần hiệu chỉnh, bảng câu hỏi cuối cùng đã được xây dựng hoàn chỉnh với 16 câu hỏi và đưa vào khảo sát định lượng sinh viên của khoa Kế toán Kiểm toán tại trường đại học Công Nghiệp thành phố Hồ Chí Minh.

Thang đo được sử dung trong bảng câu hỏi là thang đo likert 5 mức độ với ý nghĩa từng mức độ như sau: 1. Hoàn toàn không đồng ý, 2. Không đồng ý, 3. Không có ý kiến, 4. Đồng ý, 5. Hoàn toàn đồng ý.

Phương pháp khảo sát 
Nhóm nghiên cứu in hơn 1.000 bảng câu hỏi. Mỗi bảng câu hỏi bao gồm 16 biến độc lập về hoạt động giảng dạy của giảng viên và 1 biến phụ thuộc là kết quả học tập dự kiến của sinh viên đạt được thông qua hoạt động giảng dạy giảng viên. Việc khảo sát sinh viên được thực hiện trực tiếp trên lớp học của 26 giảng viên thuộc 4 khoa trong khối Kinh tế cho lớp học trả lời bảng câu hỏi.

Để đánh giá chất lượng giảng dạy của giảng viên khối Kinh tế của IUH, bài báo đã thực hiện cuộc khảo sát 926 sinh viên thuộc 4 khoa Kế toán - kiểm toán, Quản trị kinh doanh, Tài chính nhân hàng và Thương mại du lịch của IUH. Thời gian thực hiện cuộc khảo sát từ tháng 10 năm 2017 đến tháng 1 năm 2018. Số lượng phiếu khảo sát phát ra 926 phiếu, số lượng phiếu thu về 926 phiếu trong đó có 24 phiếu không hợp lệ, còn lại 902 phiếu hợp lệ. Kết quả khảo sát các khoa Kinh tế tại IUH thu được như sau. Số bảng câu hỏi đạt yêu cầu là 902.

\section{Kết quả}

Sau khi phân tích kết quả khảo sát bằng phần mềm thống kê, kết quả cho thấy mức độ hài lòng của sinh viên đối với hoạt động giảng dạy trên lớp của giảng viên các khoa kinh tế IUH ở mức cao từ với trung bình nằm trong khoảng 4.12 đến 4.50 .

Bảng 1: Mức độ hài lòng về hoạt động giảng dạy theo khoa.

\begin{tabular}{lcccccc}
\hline $\begin{array}{l}\text { Kết quả học } \\
\text { học tập mong đợi }\end{array}$ & Khoa & KTKT & QTKD & TCNH & TMDL & CHUNG \\
\hline $\begin{array}{l}\text { Hoạt động giảng dạy của giảng } \\
\text { viên }\end{array}$ & 4.42 & 4.26 & 4.31 & 4.25 & 4.32 \\
\hline
\end{tabular}

Nguồn: tác giả

Mức độ hài lòng về hoạt động giảng dạy của giảng viên khối Kinh tế ở mức cao 4.32. Mức độ hài lòng về hoạt động giảng dạy của giảng viên theo từng khoa được thể hiện như bảng 1 ở trên. Nhìn chung mức độ hài lòng của từng khoa ở mức cao từ 4.25 đến 4.42 . Trong đó cao nhất là ở khoa Kế toán - Kiểm toán ở mức 4.42, thấp nhất là khoa Thương mại du lịch ở mức 4.25. Chi tiết mức độ hài lòng của sinh viên theo theo từng hoạt động và theo giảng viên được thể hiện ở bảng 2.

Bảng 2: Mức độ hài lòng của sinh viên theo hoạt động giảng dạy của giảng viên theo khoa

\begin{tabular}{|c|c|c|c|c|c|}
\hline $\begin{array}{l}\text { Hoạt động giảng } \\
\text { dạy của giảng viên }\end{array}$ & KTKT & QTKD & TCNH & TMDL & CHUNG \\
\hline Điểm danh lớp học hàng ngày (C1.1) & 4.38 & 4.22 & 4.44 & 4.46 & 4.33 \\
\hline Kiểm tra bài cũ (C1.2) & 4.07 & 3.56 & 3.86 & 3.48 & 3.77 \\
\hline Giới thiệu mục tiêu bài học rõ ràng (C1.3) & 4.51 & 4.40 & 4.41 & 4.32 & 4.43 \\
\hline Tương tác với sinh viên khi giảng bài (C1.4) & 4.53 & 4.45 & 4.44 & 4.46 & 4.48 \\
\hline $\begin{array}{l}\text { Tóm lược bài học khi kết buối giảng dạy } \\
\text { (C1.5) }\end{array}$ & 4.42 & 4.34 & 4.31 & 4.25 & 4.36 \\
\hline $\begin{array}{l}\text { Sử dụng tốt các thiết bị công nghệ để hỗ trợ } \\
\text { cho việc giảng dạy (C1.6) }\end{array}$ & 4.40 & 4.29 & 4.33 & 4.22 & 4.33 \\
\hline $\begin{array}{l}\text { Giao bài tập về nhà cụ thể và bài đọc cho } \\
\text { buổi học kế tiếp (C1.7) }\end{array}$ & 4.42 & 4.09 & 4.23 & 4.11 & 4.23 \\
\hline $\begin{array}{l}\text { Giới thiệu tài liệu tham khảo chi tiết về từng } \\
\text { chủ đề (C1.8) }\end{array}$ & 4.26 & 4.06 & 4.14 & 4.09 & 4.14 \\
\hline $\begin{array}{l}\text { Giải đáp thắc mắc của sinh viên ngay tại lớp } \\
\text { (C1.9) }\end{array}$ & 4.54 & 4.37 & 4.46 & 4.30 & 4.44 \\
\hline $\begin{array}{l}\text { Giải đáp thắc mắc của sinh viên sau giờ học } \\
\text { (thông qua e-mail/diễn đàn } S V \text { hoặc gặp trực } \\
\text { tiếp với } G V \text {, các hình thức khác) (C1.10) }\end{array}$ & 4.20 & 4.08 & 4.04 & 4.06 & 4.12 \\
\hline $\begin{array}{l}\text { Phương pháp truyền đạt rõ ràng, dễ hiểu } \\
\text { (C1.11) }\end{array}$ & 4.39 & 4.36 & 4.33 & 4.36 & 4.37 \\
\hline Có kiến thức chuyên môn tốt (C1.12) & 4.58 & 4.43 & 4.49 & 4.46 & 4.50 \\
\hline
\end{tabular}




\begin{tabular}{|c|c|c|c|c|c|}
\hline $\begin{array}{l}\text { Hoạt động giảng } \\
\text { dạy của giảng viên }\end{array}$ & KTKT & QTKD & TCNH & TMDL & CHUNG \\
\hline $\begin{array}{l}\text { Có sự liên hệ giữa lý thuyết và thực tiễn } \\
\text { (C1.13) }\end{array}$ & 4.43 & 4.46 & 4.29 & 4.46 & 4.43 \\
\hline Đảm bảo giờ lên lớp theo quy định (C1.14) & 4.58 & 4.38 & 4.41 & 4.33 & 4.45 \\
\hline Đánh giá kết quả học tập chính xác (C1.15) & 4.50 & 4.31 & 4.36 & 4.30 & 4.38 \\
\hline Đánh giá kết quả học tập công bằng (C1.16) & 4.54 & 4.32 & 4.36 & 4.29 & 4.41 \\
\hline
\end{tabular}

Nguồn: tác giả Từ kết quả trên cho thấy mức độ hài lòng theo từng hoạt động của các khoa trong khối Kinh tế của IUH ở mức tương đối cao. Trong đó mức độ hài lòng của khoa KTKT là cao và đồng đều, còn ở các khoa khác biến độc lập "Kiểm tra bài cũ" mức độ hài lòng chỉ ở mức tương đối cao.

Kết quả học tập mong đợi của sinh viên theo khoa được thể hiện ở bảng 3 như sau:

Bảng 3: Kết quả học tập mong đợi của sinh viên theo khoa

\begin{tabular}{|c|c|c|c|c|c|}
\hline $\begin{array}{l}\text { Kết quả học } \\
\text { học tập mong đợi }\end{array}$ & KTKT & QTKD & TCNH & TMDL & CHUNG \\
\hline Điểm thường kỳ (C2.TK) & 3.96 & 3.82 & 4.04 & 4.39 & 3.96 \\
\hline Điểm giữa kỳ (C2.GK) & 3.89 & 3.68 & 3.94 & 4.11 & 3.83 \\
\hline Điểm cuối kỳ (C2.CK) & 3.86 & 3.78 & 3.96 & 4.16 & 3.87 \\
\hline
\end{tabular}

Nguồn: tác giả Theo kết quả học tập mong đợi của sinh viên ở các khoa, sinh viên mong đợi kết quả xắp xỉ 4 đương tương điểm mong đợi xoay quanh 8,0 .

Kết quả học tập thực tế của sinh viên theo khoa được thể hiện ở bảng 4 như sau:

Bảng 4: Kết quả học tập thực tế của sinh viên theo khoa

\begin{tabular}{|c|c|c|c|c|c|}
\hline $\begin{array}{l}\text { Kết quả học } \\
\text { học tập thực tế đạt được }\end{array}$ & KTKT & QTKD & TCNH & TMDL & CHUNG \\
\hline Điểm thường kỳ (C2.TK) & 4.10 & 4.18 & 4.16 & 3.94 & 4.12 \\
\hline Điểm giữa kỳ (C2.GK) & 3.73 & 3.48 & 3.80 & 4.11 & 3.68 \\
\hline Điểm cuối kỳ (C2.CK) & 3.36 & 2.74 & 3.49 & 3.19 & 3.09 \\
\hline
\end{tabular}

Nguồn: tác giả Theo kết quả học tập thực tế của sinh viên ở các khoa, điểm thực tế thường kỳ cao (trung bình 4.12) cao hơn sinh viên mong đợi (trung bình 3.96) còn lại điểm giữa kỳ và cuối kỳ thực tế đều thấp hơn mong đợi của sinh viên, tương ứng $3.68,3.09$ so với $3.83,3.87$. Đặc biệt điểm cuối kỳ thực tế thấp hơn nhiều so với kỳ vọng của sinh viên.

Để xem xét mối quan hệ giữa mức độ hài lòng và kết quả đạt được của sinh viên, bài báo phân tích mức độ hài lòng theo từng giảng viên và so sánh với kết quả học tập mong đợi và thực tế của sinh viên. Kết quả phân tích thể hiện ở bảng 5 như sau:

Bảng 5: So sánh mức độ hài lòng và điểm trung bình

\begin{tabular}{lcccc}
\hline Giảng viên & $\begin{array}{c}\text { Mức độ hài } \\
\text { lòng }\end{array}$ & $\begin{array}{c}\text { Điểm trung bình } \\
\text { chung mong đợi } \\
\text { (TBMĐ) }\end{array}$ & $\begin{array}{c}\text { Điểm trung bình } \\
\text { chung thực tế } \\
\text { (TBTT) }\end{array}$ & $\begin{array}{c}\text { Chênh lệch } \\
\text { (TBTT - } \\
\text { TBMĐ) }\end{array}$ \\
\hline GV 4 & 3.89 & 2.70 & 3.40 & 0.7 \\
GV 21 & 3.93 & 4.05 & 3.79 & -0.26 \\
GV 7 & 3.98 & 4.34 & 3.67 & -0.67 \\
GV 11 & 3.98 & 3.77 & 3.57 & -0.2 \\
GV 18 & 4.14 & 4.17 & 3.69 & -0.47 \\
GV 22 & 4.14 & 4.34 & 3.43 & -0.91
\end{tabular}




$\begin{array}{lcccc}\text { GV 13 } & 4.16 & 3.97 & 3.31 & -0.66 \\ \text { GV 20 } & 4.16 & 4.18 & 3.69 & -0.49 \\ \text { GV 15 } & 4.20 & 4.15 & 4.45 & 0.3 \\ \text { GV 6 } & 4.23 & 3.60 & 3.53 & -0.07 \\ \text { GV 19 } & 4.27 & 3.39 & 2.52 & -0.87 \\ \text { GV 16 } & 4.30 & 3.32 & 2.36 & -0.96 \\ \text { GV 25 } & 4.33 & 3.72 & 3.27 & -0.44 \\ \text { GV 10 } & 4.36 & 4,00 & 3.99 & -0.01 \\ \text { GV 12 } & 4.36 & 4.18 & 3.50 & -0.67 \\ \text { GV 23 } & 4.36 & 4.28 & 3.25 & -1.03 \\ \text { GV 26 } & 4.38 & 4.54 & 3.74 & -0.8 \\ \text { GV 9 } & 4.40 & 3.94 & 3.81 & -0.13 \\ \text { GV 3 } & 4.44 & 3.76 & 3.50 & -0.25 \\ \text { GV 5 } & 4.48 & 3.78 & 3.61 & -0.17 \\ \text { GV 17 } & 4.6 & 4.34 & 4.33 & -0.02 \\ \text { GV 1 } & 4.61 & 3.96 & 3.56 & -0.39 \\ \text { GV 14 } & 4.63 & 4.13 & 4.00 & -0.12 \\ \text { GV 8 } & 4.67 & 4.23 & 3.5 & -0.73 \\ \text { GV 2 } & 4.71 & 3.84 & 3.59 & -0.25 \\ \text { GV 24 } & 4.72 & 4.5 & 3.15 & -1.35 \\ \text { Chung } & 4.32 & 3.88 & 3.47 & (0.40)\end{array}$

Nguồn: tác giả

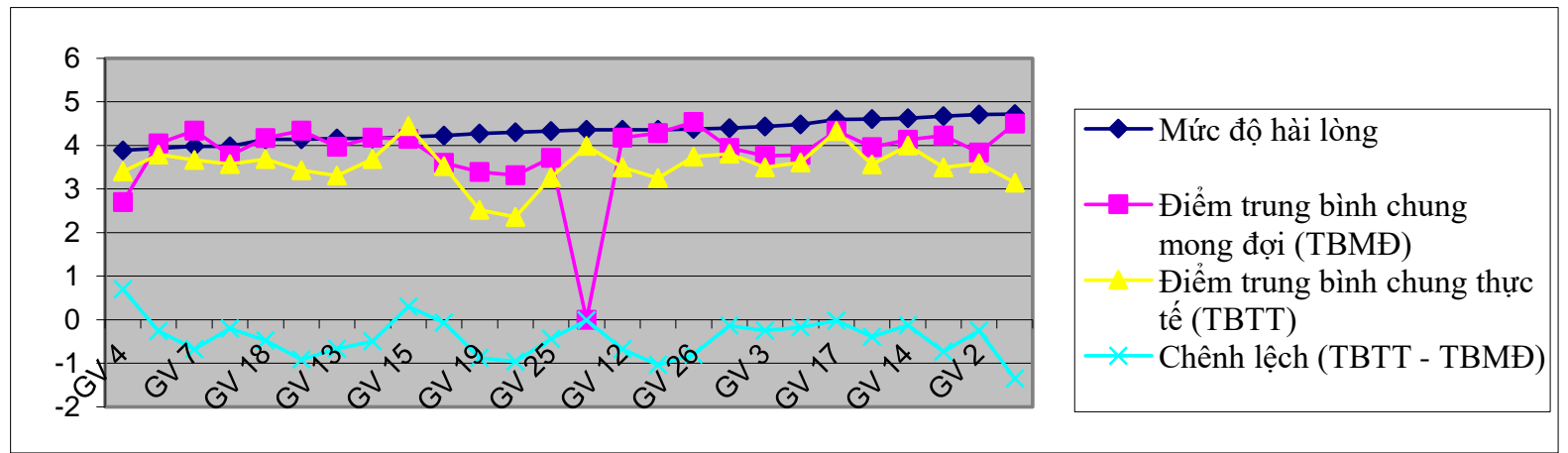

Hình 1: So sánh mức độ hài lòng và điểm trung bình

Nguồn: tác giả

\section{Bàn luận}

Theo kết quả khảo sát mức độ hài lòng của sinh viên về hoạt động "Kiểm tra bài cũ”" ở các khoa có mức độ hài lòng thấp. Các giảng viên cần chú ý nhân tố này để cải thiện hơn mức độ hài lòng của sinh viên. Nhóm nghiên tìm hiểu các khoa đều có mục "Kiểm tra bài cũ" trong kế hoạch giảng dạy nhưng các giảng viên thực hiện không đầy đủ khi lên lớp và các khoa chưa có cơ chế giám sát đánh giá để cải thiện.

Theo bảng 5 và hình 1 cho kết quả điểm trung bình thực tế của sinh viên hầu hết thấp hơn điểm trung bình mong đợi của sinh viên chỉ có 2 trường hợp $\mathrm{GV} 4$ và $\mathrm{GV} 15$ có kết quả thực tế cao hơn mong đợi của sinh viên. Thêm vào đó so sánh mức độ hài lòng của sinh viên và điểm trung bình chung thì điểm điểm trung bình chung thấp hơn mức độ hài lòng một bậc. Theo mô hình Laura Goe 2007 những giảng viên có kết quả điểm kiểm tra của sinh viên thấp hơn kết quả mong đợi đó là sự không thành công của giảng viên.

\section{Kết luận}

Nghiên cứu đã đưa ra một cái nhìn sâu sắc có giá trị về chất lượng giảng dạy mà sinh viên mong muốn từ giảng viên. Kết quả nghiên cứu cho thấy sinh viên hài lòng với hoạt động giảng dạy của giảng viên ở mức cao. Nghiên cứu này cũng cho thấy rằng sinh viên đánh giá kiến thức của giảng viên ở mức cao nhất ở mức 
4.50, mức độ hài lòng thấp nhất là "Kiểm tra bài cũ” chỉ ở mức 3.77. Các nhân tố còn lại nằm trong khoảng từ 4.12 đến 4.48 .

Nghiên cứu chỉ ra kết quả học tập thực tế của sinh viên chưa đạt được như kỳ vọng của sinh viên, đây chính là sự không thành công trong nhân tố đánh giá chất lượng giảng dạy của giảng viên. Kết quả này giúp cho các khoa trong khối ngành Kinh tế của IUH có giảng pháp nâng cao hơn nữa hoạt động giảng dạy để đáp ứng được sự mong đợi của sinh viên.

Nghiên cứu cũng chỉ ra sinh viên mong muốn giảng viên có kiến thức, nhiệt tình, dễ tiếp cận và thân thiện. Sinh viên cũng mong muốn giảng viên truyền tải những kinh nghiệm giảng dạy quý báu để có thể vượt qua các bài kiểm tra và chuẩn bị cho nghề nghiệp của mình trong tương lai.

\section{LỜI CẢM ƠN}

Chúng tôi muốn gửi lời cảm ơn tới Ban Giám Hiệu IUH, các trưởng khoa khối ngành Kinh tế của IUH đã tạo điều kiện cho chúng tôi thực hiện nghiên cứu này. Chúng tôi cũng gởi lời cảm ơn tới các đồng nghiệp và những người cộng tác. Sau cùng chúng tôi cảm ơn đến tất cả các tác giả mà chúng tôi trích dẫn trong bài báo.

\section{TÀI LIỆU THAM KHẢO}

1. Nguyễn Thị Thu Trang, tự chủ đại học và vấn đề đánh giá chất lượng giảng viên đại học. http://vienphuongdong-ordi.vn/tu-chu-dai-hoc-va-van-de-danh-gia-chat-luo\%CC\%A3nggia\%CC\%89ng-vien-da\%CC\%A3i-ho\%CC\%A3c/

2. Thi Lan Phuong Pham, 2012. The Renovation of Higher Education Governance in Vietnam and its Impact on the Teaching Quality at Universities, Tertiary Education and Management, 18 (4), 289-308.

3. Laura Goe, 2007. The link between teacher quality and student outcomes: A research synthesis. Washington, DC: National Comprehensive Center for Teacher Quality. https://files.eric.ed.gov/fulltext/ED521219.pdf

4. Lê Đình (2008). Đánh giá giảng dạy - Một nhân tố quan trọng trong đảm bảo và nâng cao chất lượng giáo dục đại học. http://www.ussh.vnu.edu.vn/d6/vi-VN/news/

5. Harry G. Murray, 1984. The impact of formative and summative evaluation of teaching in north American Universities. Assessment \& Evaluation in Higher Education, 9(2), 117-132.

6. Suryaman, 2018. Indonesian Private University Lecturer Performance Improvement Model to Improve a Sustainable Organization Performance. International Journal of Higher Education, 7(1).

7. Xiao, J. and Wilkins, S, 2015. The effects of lecturer commitment on student perceptions of teaching quality and student satisfaction in Chinese higher education. Journal of Higher Education Policy and Management, 37(1), 98-110.

8. Jacqueline Douglas \& Alex Douglas, 2006. Evaluating Teaching Quality, Quality in Higher Education, 12(1), 3-13.

9. Drs. H. Akbar Ali, M.Si, 2015. Performance lecturer's competence as the quality assurance. The International Jounal of Social Sciences, 30(1).

Ngày nhận bài: 05/10/2018

Ngày chấp nhận đăng: 06/12/2019 